\title{
Uncertainties in the Quantification of Supraharmonic Emission: Variations over Time
}

A. Espín-Delgado ${ }^{1}$, S. K. Rönnberg ${ }^{1}$ and M. H. J. Bollen ${ }^{1}$

\author{
${ }^{1}$ Electric Power Engineering Group \\ Luleå University of Technology \\ Campus Skellefteå, 93187 Skellefteå (Sweden) \\ e-mail: angela.espin.delgado@ltu.se
}

\begin{abstract}
This paper shows a study of the variations of the supraharmonic distortion emitted by an LED lamp during and after its thermal stabilization. The study is aimed to contribute to map the uncertainties existing when quantifying supraharmonics. Measurements of the current drawn by five lamps of the same type and manufacturer are performed. Then, analysis in the frequency domain is performed and the Total Supraharmonic Current (TSHC) index is used to quantify the supraharmonic emission of the lamps. The TSHC is calculated over bands of different widths, whose outcomes are then compared to decide which bandwidth results in a less time-varying-TSHC index. It is demonstrated that the supraharmonic distortion emitted by the lamp under study experiences both an increase in magnitude and a shift in frequency during the thermal stabilization of the lamp. It is also shown how the frequency grouping impacts the quantification. It is concluded that the best choice of a grouping bandwidth is one wide enough so that it includes the whole emission band of interest. This result is a step forward to set up references for the analysis and quantification of broadband supraharmonic emission.
\end{abstract}

Key words. Supraharmonics, LED, power quality, highfrequency distortion, efficiency lighting

\section{Introduction}

Supraharmonics are voltage and current waveform distortion in the frequency range 2 to $150 \mathrm{kHz}$. The study of the impact of supraharmonics on low-voltage networks have received increasing attention during the last decade [1] due to the increasing amount of devices utilizing switching frequencies between 2 and $150 \mathrm{kHz}$ as well as the growing usage of narrowband PLC (Power Line Communication).

Levels of immunity to supraharmonics and compatibility levels for voltage distortion in the supraharmonic range can be found in the standards IEC 61000-4-19 and IEC 61000-2-2, respectively. Methods for measuring supraharmonics are available in IEC 61000-4-7 and IEC 61000-4-30. However, the definition of limits for emission of supraharmonics is still under discussion and research is ongoing on the matter. The assessment of how much distortion a bulk use of power electronics devices can inject into the grid is necessary before stating emission limits and planning levels for supraharmonics. To address this issue, the first step is to characterize the supraharmonic emission from individual devices considering relevant parameters that represent the distortion as well as the uncertainties that might affect the models.
It has been shown in the literature that lighting emitting diode (LED) lamps emit more or less distortion in the supraharmonic range depending on their drivers' topology [2], [3], [7]. The characteristics of the supraharmonic emission from LED lamps have been presented in previous works: in [7], the grouping of frequency components over the whole supraharmonic range is used to quantify the distortion; in [6], the highest component over a frequency range is used. The study presented in this paper concerns the quantification of the supraharmonic emission of LED lamps considering uncertainties.

Since one source of uncertainty when quantifying supraharmonics is how they vary over time, the present work is aimed to observe: 1) the behavior of the supraharmonic distortion emitted by the lamps during their thermal stabilization, and 2) the variations of the supraharmonic distortion after thermal stabilization. The study specifically brings up the discussion about how grouping in the supraharmonic range, as it is recommended by IEC [9], affects the quantification results. It is demonstrated that the supraharmonic emission of the lamps under study presents variations both during and after thermal stabilization. How much these variations affect the quantification of the emission depends on the grouping bandwidth used.

This result contributes to the practices on the analysis of broadband supraharmonic emission. Apart from LED lamps, other appliances are emitters of this type of distortion, e.g., electric vehicles and computers. The contributions achieved are a step forward to obtain references and achieve guidelines on how to analyze and quantify supraharmonic emission in low-voltage networks, as they are challenges acknowledged by experts in the power quality field [10], [11].

\section{Experimental Procedure}

The experimental procedure used to obtain the data to be analyzed is explained below.

\section{A. Experiment Setup}

The equipment under test is the LED lamp, $12 \mathrm{~W}$ nominal power, $806 \mathrm{~lm}$ luminous flux and $2700 \mathrm{~K}$ temperature color, 
fitted with an active power factor correction (APFC) feature. A picture of the lamp under test is shown in Fig. 1a. Ten LED lamps of the same type and manufacturer (Fig. 1a) were connected in parallel and supplied with a sinusoidal voltage through a voltage amplifier configuration as is depicted in Fig. 1b. Detailed information about the amplifier configuration can be found in [4]. The lamp shadowed in blue in Fig. $1 \mathrm{~b}$ is named from now on as the reference lamp.
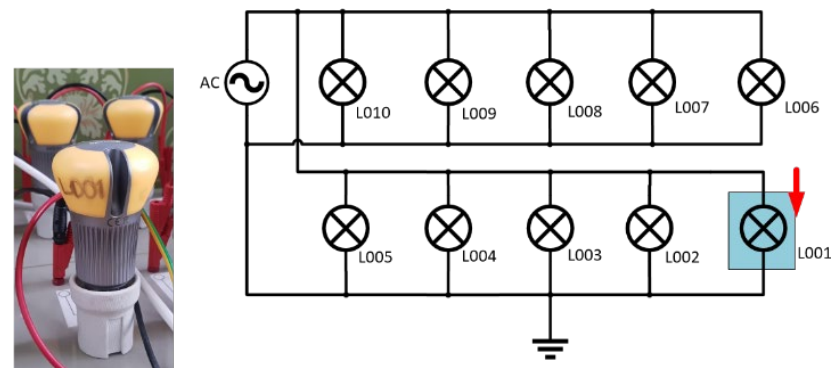

Fig. 1a. LED lamp Fig. 1b. Experiment setup diagram.

Fig. 1. Lamp under test and setup diagram.

A Hioki oscilloscope is used to measure the supply voltage of the installation and the current of the reference lamp, with a sampling frequency of $10 \mathrm{MHz}$. The red arrow in Fig. $1 \mathrm{~b}$ represents the measured current. Fig. 2 shows the waveform of the voltage and the reference lamp current during one cycle. It is seen that the lamp current contains supraharmonic distortion during most of the time lapse. There is no supraharmonic distortion around the voltage zero-crossing as no current is drawn by the lamp at that instance. This paper is focused on the frequency domain so no further comments will be made about the time domain characteristics of the signals.

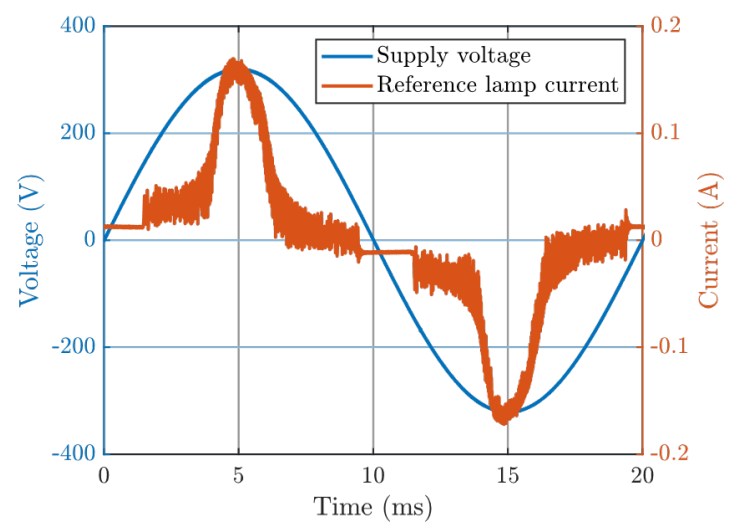

Fig. 2. Voltage supplied and current drawn by the lamp during one cycle.

In order to observe the frequency components of this supraharmonic distortion, the spectrum of the current signal in the supraharmonic range is presented in Fig 3. The spectrum was calculated by applying the $f f t$ function to 10 cycles $(200 \mathrm{~ms})$ of the current in Matlab, giving a $5 \mathrm{~Hz}$ resolution. The highest components of supraharmonic distortion of the current appear as concentrated around 45 $\mathrm{kHz}$. This highly supraharmonic polluted frequency band will be named from now on as the main supraharmonic emission of the lamp.

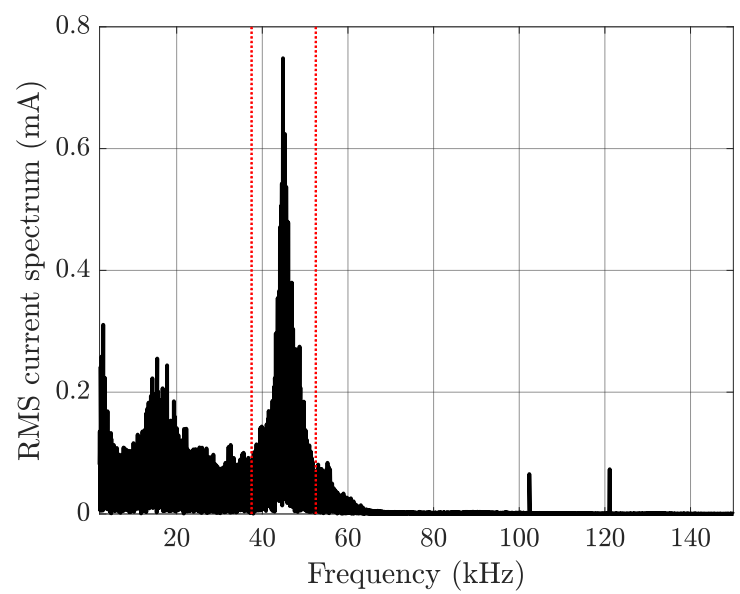

Fig. 3. Supraharmonic spectrum of the lamp L001 current, $5 \mathrm{~Hz}$ resolution.

\section{B. Experiment Method}

The experiment is aimed to observe: 1) the behavior of the supraharmonic distortion emitted by the lamps during their thermal stabilization, and 2) the variations of the supraharmonic distortion after thermal stabilization which leads to uncertainties in further analysis. This implies the measurement of the lamp current repeatedly and systematically after the lamp had been turned on. Measurements and subsequent recordings of 10 cycles (200 $\mathrm{ms}$ ) of the current waveform were performed every 5 minutes starting from the instant where the supply of the installation was switched on. The previously explained procedure will be labeled from now on as "a set of measurements".

The experiment consists in performing a set of measurements five times. For every set of measurements, the reference lamp was changed for another individual of the same type. This results in a set of measurements for every lamp from L001 to L005. The first set of measurements were taken while the lamp L001 was playing the role of reference lamp; the second set of measurements corresponded with the lamp L002 being the reference lamp and so on.

Approximately the first six hours of operation (200 ms every five minutes) were recorded for lamp L001. Approximately two hours of operation were recorded for lamps L002 to L005. A cooling down period of at least three hours was considered before every new set of measurements was taken.

\section{Thermal Stabilization Time}

A clear explanation of the concept of thermal stabilization time can be found in [4]. The thermal stabilization time of the lamp under study has been investigated in [4], [5] from different approaches. The lamp in Fig. 1a is labelled as LED 03 in [4], and as LED 6 in [5].

In both works, the authors measure and analyze a characteristic variable of the lamp as a function of the operation time (after switching on), $\boldsymbol{\Delta} \boldsymbol{M}\left(\boldsymbol{t}_{\boldsymbol{n}}\right)$. The criterion that determines the thermal stabilization time is based on the difference between two consecutive measurements, $\boldsymbol{\Delta} \boldsymbol{M}\left(\boldsymbol{t}_{\boldsymbol{n}}\right)$, and a threshold, $\boldsymbol{c}$. In summary, the lowest time value, $\boldsymbol{t}_{\boldsymbol{n}}$, for which (1) holds defines the thermal 
stabilization time, $\boldsymbol{t}_{\boldsymbol{n}}$. The difference between the criteria in [4] and [5] is summarized in Table I.

$$
\Delta M\left(t_{n}\right) \leq c
$$

Table I. Criteria for thermal stabilization time

\begin{tabular}{|l|c|c|c|}
\hline Criterion & $\begin{array}{c}\text { Variable } \\
\boldsymbol{M}\end{array}$ & $\begin{array}{c}\text { Threshold } \\
\boldsymbol{c}\end{array}$ & $\begin{array}{c}\text { Time between } \\
\text { measurements } \\
\boldsymbol{t}_{\mathbf{2}}-\boldsymbol{t}_{\mathbf{1}}\end{array}$ \\
\hline $\begin{array}{l}\text { Sakar et } \\
\text { al. }\end{array}$ & Light intensity & $0.5 \%$ & $15 \mathrm{~min}$ \\
\hline $\begin{array}{l}\text { Gutiérrez } \\
\text { et al. }\end{array}$ & Temperature & $1 \%$ & $10 \mathrm{~min}$ \\
\hline
\end{tabular}

The thermal stabilization time of the lamp under study (Fig. 1a) is then reported as 60 and 70 minutes in [4] and [5], respectively, when supplied with sinusoidal voltage.

The RMS value of the fundamental current of every tested lamp during the first $110 \mathrm{~min}$ of operation is plotted in Fig. 4. The fundamental current decreases rapidly during the first $20 \mathrm{~min}$ of operation and stabilizes around $60 \mathrm{~min}$ following the trend experienced by the light intensity and the temperature reported in [4] and [5], respectively.

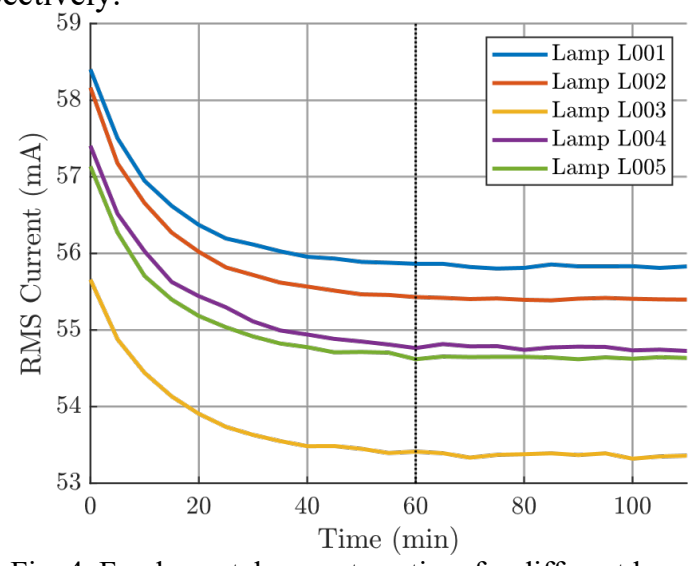

Fig. 4. Fundamental current vs. time for different lamp individuals.

\section{Analysis of Supraharmonic Distortion}

This section describes the method to be used for quantifying the supraharmonic emission during the thermal stabilization of the lamps. Moreover, the impact of using different frequency grouping bandwidths in the quantification of supraharmonics is evaluated and discussed. The procedure for the analysis in the frequency domain is summarized in steps as described next:

A. Defining a center frequency of the supraharmonic emission: From Fig. 4, it can be deduced that every individual lamp, even though they are of the same type and manufacturer, has intrinsic characteristics that differentiate them from the others. Following this reasoning, it can be deduced that the supraharmonic emission of every lamp is also slightly different for each one of them.

The supraharmonic frequency spectrum (see Fig. 3 ) of the current after thermal stabilization ( $t \geq 60 \mathrm{~min}$ ) is inspected so that a center frequency of the main supraharmonic emission is identified for every individual lamp studied as shown in Table II. Taking advantage of the symmetrical characteristic of the main supraharmonic emission of this lamp, as appears in the frequency domain, it is possible to select the highest $5 \mathrm{~Hz}$ frequency component as the central one. Further work is required regarding the characterization of broadband supraharmonics in case of, for example, asymmetry in the frequency domain representation. An initial approach would be to define the grouping borders in terms of a certain signal-to-noise ratio SNR $>x$ and determine the middle as the center frequency.

Table II. Center frequency of supraharmonic emission

\begin{tabular}{|l|c|c|c|c|c|}
\hline Lamp & L001 & L002 & L003 & L004 & L005 \\
\hline $\begin{array}{l}\text { Center frequency } \\
(\mathrm{kHz})\end{array}$ & 45 & 44 & 45.7 & 45.4 & 45.7 \\
\hline
\end{tabular}

B. Defining different grouping bandwidths: Only the main supraharmonic emission of the lamp is to be studied in this paper. In order to avoid the influence of noise outside the main emission range, a grouping of the components around this main emission will be performed considering different bandwidths. The frequency grouping bandwidths to be used in the analysis and compared to each other are 2, 5, 10, 15 and $20 \mathrm{kHz}$. The $2 \mathrm{kHz}$ grouping has been chosen such that it is in accordance with the bandwidth recommended in IEC 61000-4-30, Annex C [9] for frequencies between $9 \mathrm{kHz}$ and $150 \mathrm{kHz}$. The wider grouping bandwidths are proposed after observing in Fig. 3 that the main emission is certainly broader than $2 \mathrm{kHz}$ (the red dotted lines in the figure represent the boundaries of a $15 \mathrm{kHz}$ wide band centered at $45 \mathrm{kHz}$ ). The center frequency is kept fixed for all the bands and varies only with the individual (see Table II).

C. Calculating the TSHC: The Total Supraharmonic Current (TSHC) index was proposed by Grevener in [8], [12] for quantifying the supraharmonic emission over a frequency range. This index is based on Parseval's theorem [13] and has been used in other publications for analysis of supraharmonic distortion [2]. The TSHC is defined as follows:

$$
\mathrm{TSHC}=\sqrt{\sum_{s=f_{\min }}^{f_{\max }} I_{s}^{2}}
$$

where $s$ is the index of the RMS current value, $I$, of the frequency component starting in the lower frequency border of a grouping band, $f_{\text {min }}$, and ending in the upper frequency border of a grouping band $f_{\max }$.

\section{Supraharmonic Distortion during Thermal Stabilization Time}

This section summarizes the results obtained after applying the analysis procedure explained above to the measurements recorded during thermal stabilization time of the lamps.

The TSHC is calculated for every 10-cycles measurement based on five different frequency bands. Fig. 5 shows the calculated TSHC as a function of time for lamp 
L001. The supraharmonic distortion emitted by the lamps will be treated from now on using absolute quantities (mA). In general, an increasing tendency of the TSHC magnitude can be observed in Fig. 5 during the first hour of operation of the lamp for all the grouping bands evaluated. After this first hour, the TSHC experiences small variations around a constant average value. These variations will be quantified in section 5. The same $60 \mathrm{~min}$ pattern in the TSHC is observed for lamps L002 to L005, whose results are not reproduced here due to space limitations. These results lead to think that the supraharmonic distortion emitted by the lamp changes during its thermal stabilization time but further analysis will be done before concluding so.

It is also important noticing, in Fig. 5, the difference between the TSHC values concerning different grouping bandwidths. The TSHC increases as wider is the bandwidth. The 10, 15 and $20 \mathrm{kHz}$ grouping bandwidths yield similar final values of TSHC (around $7.5 \mathrm{~mA}$ after 6 hours of continuous operation of the lamp), being $3.5 \%$ the difference between the last point of the green curve and the yellow curve in Fig. 5. The similarities between the 10, 15 and $20 \mathrm{kHz}$ grouping curves and their evolution over time suggest that the difference between them is essentially due to the components being added as the bandwidth gets wider.

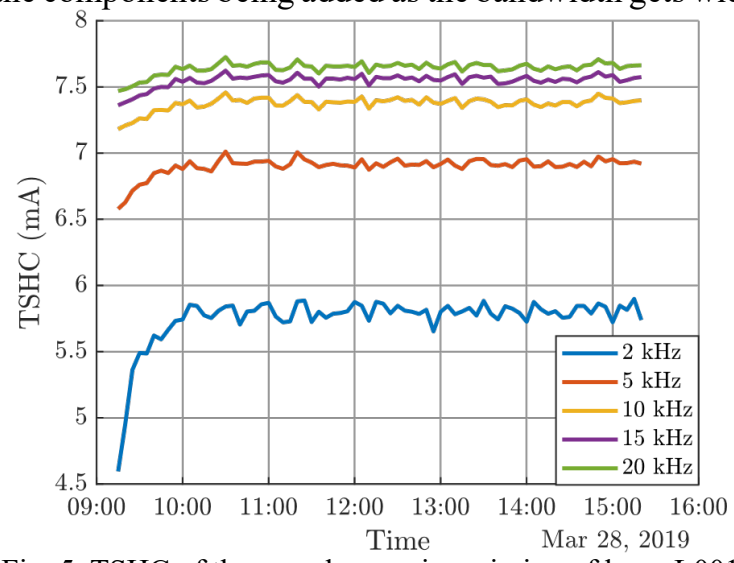

Fig. 5. TSHC of the supraharmonic emission of lamp L001 calculated based on different bandwidths.

In comparison with the three cases mentioned above, the curve of the TSHC being calculated using a $2 \mathrm{kHz}$ bandwidth (blue curve in Fig. 5) shows a steeper increase during the first $60 \mathrm{~min}$ of continuous operation of the lamp. This characteristic will be explained further later on. The 2 $\mathrm{kHz}$-grouping-TSHC after $6 \mathrm{~h}$ of operation of the lamp is $5.7 \mathrm{~mA} ; 24 \%$ lower than when the TSHC is calculated using a $15 \mathrm{kHz}$ bandwidth. The latter indicates that there are some supraharmonic components in the frequency domain that are not taken into account when using a grouping band as narrow as $2 \mathrm{kHz}$. Fig. 3 shows that the main supraharmonic emission is indeed broader than $2 \mathrm{kHz}$.

In Fig. 5, the increase of the TSHC calculated using a $5 \mathrm{kHz}$ grouping bandwidth over the first hour of operation of the lamp is not as steep as the $2 \mathrm{kHz}$-grouping case but certainly steeper than the other cases.

A better comparison of the five methods for calculating the TSHC is achieved if relative values are shown. Fig. 6 presents the TSHC over time relative to the first calculated value for each case. The curves corresponding with 10,15 and $20 \mathrm{kHz}$-bandwidth calculations present similar behavior. The latter indicates that the differences between the corresponding curves in Fig. 5 are actually due to the inclusion of noise in the grouping process for the TSHC calculation due to the broadening of the bandwidth. Having eliminated this noise aggregation effect, what can be seen on these three almost identical curves (Fig. 6) is an actual increase of the supraharmonic distortion emitted by the lamp during the thermal stabilization period. After the thermal stabilization period, the supraharmonic emission of this lamp, quantified by the TSCH index, has increased in average 3\% with relation to when it was first turned on.

Concerning the $2 \mathrm{kHz}$ grouping and the $5 \mathrm{kHz}$ grouping calculations, the TSHC had increased in about $25 \%$ and $5 \%$ its initial value after the thermal stabilization time of the lamp. The fact that these values are higher than the $3 \%$ encountered for the three calculation cases explained before suggests that there is a phenomenon other than the actual increase in the magnitude of the supraharmonic emission impacting the calculation of the TSHC.

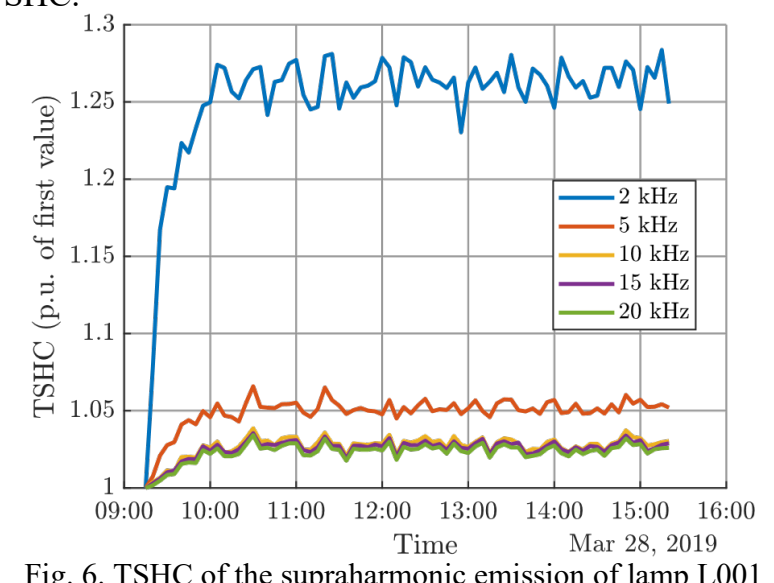

Fig. 6. TSHC of the supraharmonic emission of lamp L001 calculated using different grouping bandwidths and relative to the first TSHC value.

Fig. 7 presents the spectrum of the supraharmonic emission of lamp L001 over the $2 \mathrm{kHz}$ band used for the TSHC calculation, after 0 and $60 \mathrm{~min}$ of continuous operation. The center frequency of the main supraharmonic emission at time $t=0 \mathrm{~min}$ is clearly shifted from $45 \mathrm{kHz}$, which is the center frequency at time $t=60 \mathrm{~min}$. A clearer picture can be observed in Fig. 8, which shows the spectrum over a 15 $\mathrm{kHz}$ band. Observe that the center frequency of the main supraharmonic emission at time $t=0 \mathrm{~min}$ is located at 47 $\mathrm{kHz}$. As the lamp stabilizes thermally, the center frequency of the main supraharmonic emission in turn shifts and stabilizes at a lower frequency (in this case, $45 \mathrm{kHz}$ ). The previously explained phenomenon causes that, having a 2 $\mathrm{kHz}$ bandwidth with fixed boundaries $(44 \mathrm{kHz}$ to $46 \mathrm{kHz}$ for lamp L001), the calculation of the TSHC miss some components of the supraharmonic emission during the thermal stabilization time.

\section{Supraharmonic Distortion after Thermal Stabilization Time}

This section intends to quantify the differences between grouping methods and between individual lamps, after their thermal stabilization time, by analyzing the TSHC index. 


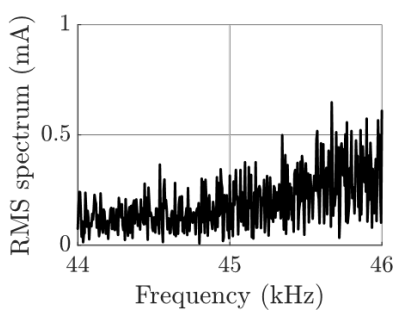

Fig. 7a. 0 min elapsed

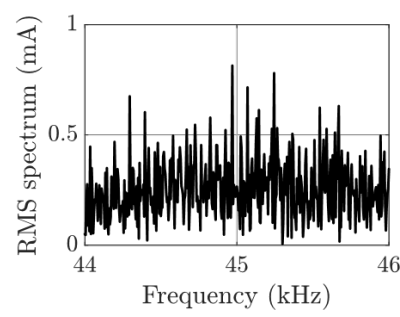

Fig. 7 b. 60 min elapsed
Fig. 7. Spectrum of the supraharmonic emission of lamp L001 over a $2 \mathrm{kHz}$ band centered at $45 \mathrm{kHz}$ after 0 and 60 min of operation.

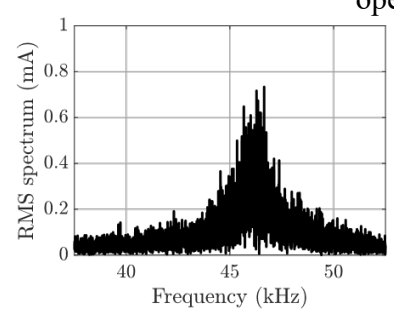

Fig. 8 a. 0 min elapsed

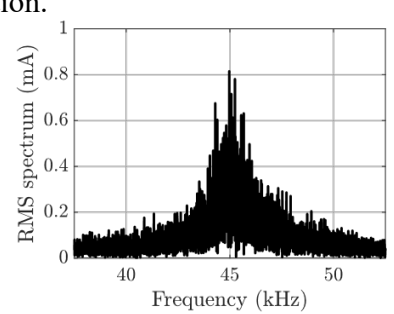

Fig. 8b. 60 min elapsed
Fig. 8. Spectrum of the supraharmonic emission of lamp L001 over a $15 \mathrm{kHz}$ band centered at $45 \mathrm{kHz}$ after 0 and $60 \mathrm{~min}$ of operation.

The averaged TSHC is used to characterize the supraharmonic distortion emitted by every lamp. This index is calculated averaging all TSHC values after the thermal stabilization time $(t \geq 60 \mathrm{~min})$ for different individuals and grouping bandwidths. The results are presented in Fig. 9.

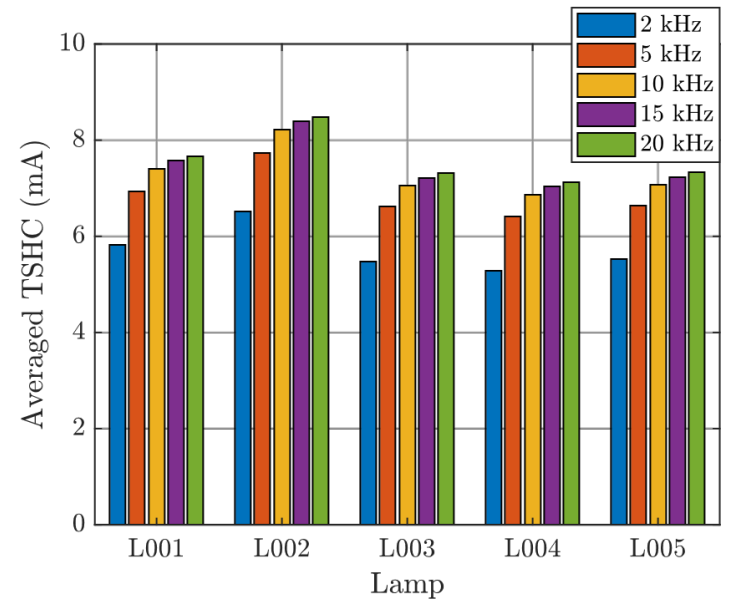

Fig. 9. Averaged TSHC after thermal stabilization time for different individuals and grouping bandwidths.

The data showed in Fig. 9 is in agreement with the results and analysis presented in section IV. For every individual, the TSHC index increases as the bandwidth broadens due to the subsequent inclusion of supraharmonic and noise components. The averaged TSHC of the distortion emitted by these lamps is between 5 and $8.5 \mathrm{~mA}$, depending on the grouping method used.

The standard deviation of the TSHC for $\mathrm{t} \geq 60 \mathrm{~min}$ is used in Fig. 10 for quantifying the variation of the distortion emitted by the lamps over time and how the grouping bandwidth selection affects this quantification. It is clear that using a $2 \mathrm{kHz}$ bandwidth leads to higher variations in the TSHC data over time. In general, a wider grouping band gives a better representation of the supraharmonics emitted by one lamp over time. The TSHC corresponding with lamp L003 presents the highest variations; the reason is unknown. One can hypothesize that it is related to aging after noticing in Fig. 4 that lamp L003 draws the lowest current ergo has the lowest power. Further work should confirm or refute this hypothesis.

Another observation from Fig. 9 is the difference between individuals in relation to their supraharmonic emission after thermal stabilization time. Although the five lamps present similar behaviors in terms of fundamental current, increase of supraharmonic distortion emitted during thermal stabilization time, and how this emission is represented in the frequency domain (shape of the spectrum in Fig. 3 and 8), they cannot be considered identical. The latter should be considered when attempting predictions of aggregated supraharmonic emissions (e.g. from a whole lighting installation) based on the emission from one device.

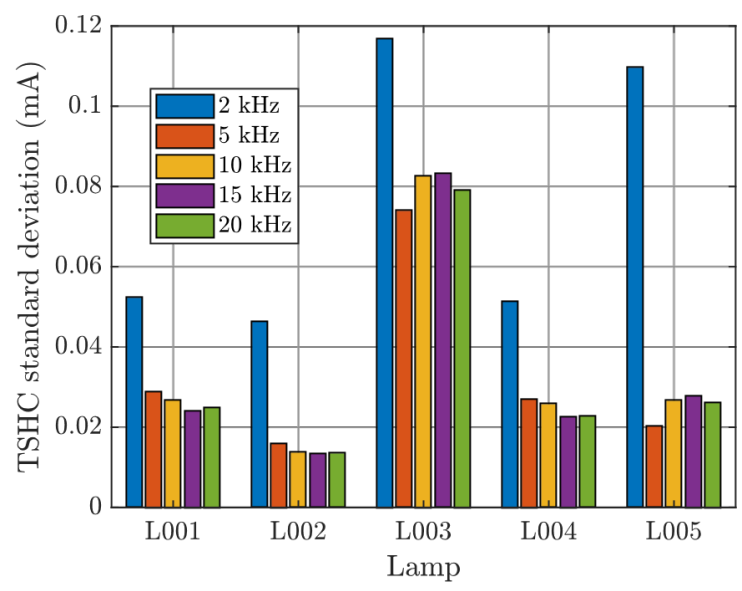

Fig. 10. Standard deviation of TSHC after thermal stabilization time for different individuals and grouping bandwidths.

An alternative representation of the variability of the TSHC calculated based on the measurements after thermal stabilization can be attained by using a box plot as in Fig. 11. On each box, the central mark indicates the median, and the bottom and top edges of the box indicate the 25 th and 75th percentiles, respectively. The whiskers extend to the most extreme data points. The data in Fig. 11 corresponds to the current of lamp L004 and includes, for reference purposes, an additional box describing the variability of the TSHC calculated based on a $200 \mathrm{~Hz}$-grouping bandwidth around the center frequency of the main supraharmonic emission of the lamp. $200 \mathrm{~Hz}$-grouping is recommended in [14] for distortion between 2 and $9 \mathrm{kHz}$.

Figure 11 shows further evidence of the suitability of choosing a broader grouping bandwidth for quantifying the main supraharmonic emission. Notice in Fig. 11 that the difference between the extreme data points, when using a $200 \mathrm{~Hz}$-grouping bandwidth for the calculation of the TSHC, is higher than using any of the other grouping bandwidths. The right-hand plot in Fig. 11 confirms the observations made about Fig. 9 and 10. These results give a general picture about the uncertainties incurred when quantifying the supraharmonic emission from an individual in the frequency domain. As an example, for lamp L004, the deviation of the TSHC over time about its mean value when using a $10 \mathrm{kHz}$-grouping bandwidth is $\pm 0.8 \%$; while 
the deviation using a $200 \mathrm{~Hz}$-grouping bandwidth is $\pm 14 \%$. Lastly, the worst uncertainty case in this experiment relates to lamp L003 (Fig. 10): the deviation of the TSHC over time about its mean value when using a $10 \mathrm{kHz}$-grouping bandwidth is $\pm 2 \%$ for this individual.

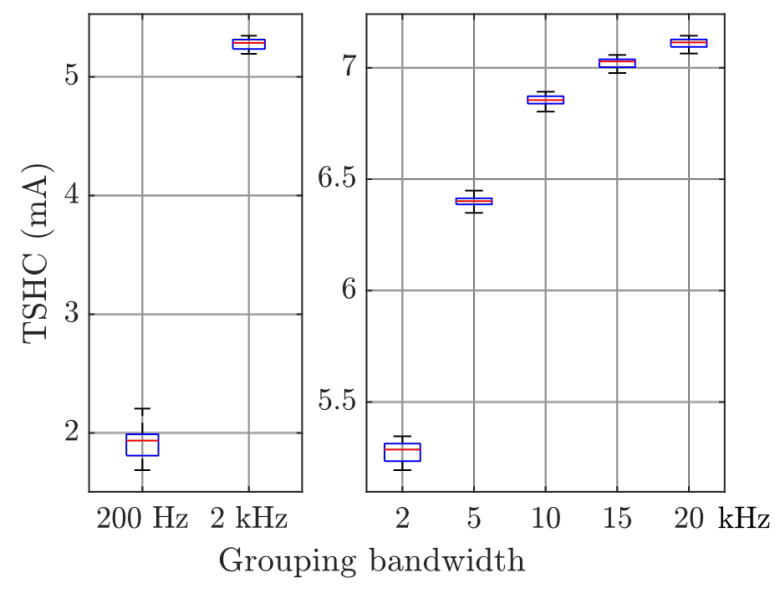

Fig. 11. Variation of TSHC of the supraharmonic emission of lamp L004 after thermal stabilization. Comparison with a 200 Hz-grouping bandwidth.

\section{Conclusions}

The conclusions of this study are summarized next:

- The thermal stabilization time of the lamp can be identified based on its fundamental current in addition to the temperature and the light output.

- The supraharmonic distortion emitted by the lamp under study experiences an increase in magnitude during the thermal stabilization of the lamp. The distortion also shifts in frequency during the thermal stabilization of the lamp. On the basis of this evidence, one can hypothesize that lamps with similar characteristics to this one (namely, lamps with drivers using APFC) behave similarly. Experiments with a variety of lamps of different manufacturers should be performed in order to generalize this point.

- The characterization of the behavior of the supraharmonic distortion emitted by this lamp during its thermal stabilization is better achieved by using a grouping bandwidth of at least $10 \mathrm{kHz}$ for calculating the TSHC. The band should be broad enough so that it involves the whole emission of interest plus possible shifts in frequency happening during the thermal stabilization of the lamp.

Although there is a correspondence between changes in the supraharmonic emission and thermal stabilization of the lamp, the supraharmonic emission presents variations even when the lamp can be considered stable. How much these variations affect the quantification of the emission depends on the bandwidth used to calculate the TSHC. Variations in the quantification of the main supraharmonic emission can be reduced by using a grouping band wide enough, e.g.: 10 and $20 \mathrm{kHz}$ give better results than using 200 $\mathrm{Hz}$ and $2 \mathrm{kHz}$ bandwidths. The highest TSHC variation after thermal stabilization, for the lamps tested in this experiment, using a $10 \mathrm{kHz}$ grouping bandwidth, is \pm 2 $\%$ of the average value of the sample data. The latter value can be used as a reference for expected deviations in the quantification of supraharmonic emission for this type of lamp.

- Standardization measures for quantification of supraharmonic emission from LED lamps should consider a stabilization time and grouping bandwidths broader than those currently used. In this case, $60 \mathrm{~min}$ and $10 \mathrm{kHz}$ are recommended.

\section{References}

[1] S. Subhani, V. Cuk, J. F. G. Coben, "A Literature Survey on Power Quality Disturbances in the Frequency Range of $2-150 \mathrm{kHz}$, Proceedings of the International Conference on Renewable Energies and Power Quality (ICREPQ'17), 2017.

[2] A. Gil-De-Castro, R. Medina-Gracia, S. K. Rönnberg, A. M. Blanco, and J. Meyer, "Differences in the performance between CFL and LED lamps under different voltage distortions," in 18th International Conference on Harmonics and Quality of Power (ICHQP), May 2018, pp. $1-6$.

[3] C. Waniek, T. Wohlfahrt, J. M. A. Myrzik, J. Meyer, M. Klatt, and P. Schegner, "Supraharmonics: Root causes and interactions between multiple devices and the low voltage grid," in 2017 IEEE PES Innovative Smart Grid Technologies Conference Europe (ISGT-Europe), Sep. 2017, pp. 1-6.

[4] S. Sakar, S. Rönnberg, and M. H. J. Bollen, "Light intensity behavior" of LED lamps within the thermal stabilization period," in 18th International Conference on Harmonics and Quality of Power (ICHQP), May 2018, pp. 1-6.

[5] E. Gutierrez-Ballesteros, A. Gil-de-Castro, S. Rönnberg, and S. Sakar, "Temperature and voltage distortion analysis in LED lamps," in 2019 25th International Conference on Electricity Distribution (CIRED), June 2019, pp. 1-5.

[6] A. M. Blanco, R. Stiegler and J. Meyer, "Power quality disturbances caused by modern lighting equipment (CFL and LED)," Power Tech Conference, Grenoble, 2013, pp. $1-6$.

[7] X. Xu, A. Collin, S. Z. Djokic, R. Langella, A. Testa, and J. Drapela, "Experimental evaluation and classification of LED lamps for typical residential applications," in 2017 IEEE PES Innovative Smart Grid Technologies Conference Europe (ISGT-Europe), Sep. 2017, pp. 1-6.

[8] A. Grevener, J. Meyer, S. Rönnberg, M. Bollen, and J. Myrzik, "Survey of supraharmonic emission of household appliances," CIRED - Open Access Proceedings Journal, vol. 2017, no. 1, pp. 870-874, 2017.

[9] IEC 61000-4-30, "Part 4-30: Testing and measurement techniques Power quality measurement methods," Electromagnetic Compatibility (EMC), vol. 3, 2015.

[10] S. K. Rönnberg, M. H. Bollen, H. Amaris, G. W. Chang, I. Y. Gu, L. H. Kocewiak, J. Meyer, M. Olofsson, P. F. Ribeiro, and J. Desmet, "On waveform distortion in the frequency range of $2 \mathrm{kHz}-150 \mathrm{kHz}$ Review and research challenges," Electric Power Systems Research, vol. 150, pp. $1-10,2017$.

[11] JWG C4.24/CIRED, "Power quality and EMC issues with future electricity networks," Technical Brochures, vol. 719, 2018.

[12] A. Grevener, "Characterization of supraharmonic emission caused by electronic equipment," Master's thesis, Technische Universität Dresden, 2016.

[13] M. H. Bollen and I. Y. Gu, Signal processing of power quality disturbances. John Wiley \& Sons, 2006, vol. 30.

[14] IEC 61000-4-7, "Part 4-7: Testing and measurement techniques General guide on harmonics and interharmonics measurements and instrumentation, for power supply systems and equipment connected thereto," Electromagnetic Compatibility (EMC), 2004. 